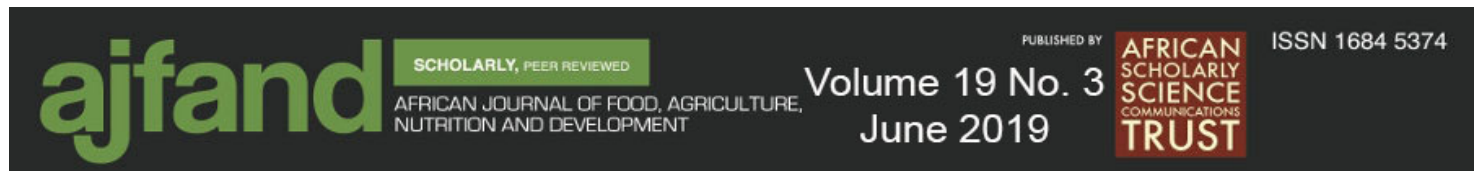

Afr. J. Food Agric. Nutr. Dev. 2019; 19(3): 14690-14707

DOI: 10.18697/ajfand.86.17380

\title{
ETUDE DES PERFORMANCES TECHNIQUES D'UN FILTRE-PRESSE POUR LA FILTRATION DU JUS DE POMME D'ANACARDE (Anacardium occidentale L.)
}

\section{Dossou $\mathbf{J}^{1^{*}}$, Ahouansou RH ${ }^{2,3}$, Sanya CAK ${ }^{1}$, V Ahyi ${ }^{4}$}

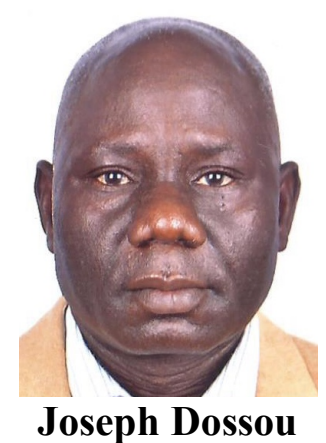

*Contact auteur correspondant: jokdossou@yahoo.fr

${ }^{1}$ Laboratoire de Bioingénierie des Procédés Alimentaires, Faculté des Sciences Agronomiques, Université d'Abomey-Calavi, 01 B.P. 526 Cotonou, Bénin

${ }^{2}$ Laboratoire d'Energétique et de Mécanique Appliquée (LEMA); Ecole Polytechnique d'Abomey-Calavi (EPAC) ; Université d'Abomey-Calavi (UAC), 01 BP:2009 Cotonou, Benin

${ }^{3}$ Programme de Technologies Agricoles et Alimentaires, Centre de Recherches Agricoles d'Agonkanmey, Institut National des Recherches Agricoles du Bénin, 01 BP 128 PortoNovo, Bénin

${ }^{4}$ Laboratoire de Diagnostics, d'Expertises Biologiques et Chimiques de l'Institut Régional du Génie Industriel des Biotechnologies et Sciences Appliquées, 07 BP 231Cotonou, Bénin 


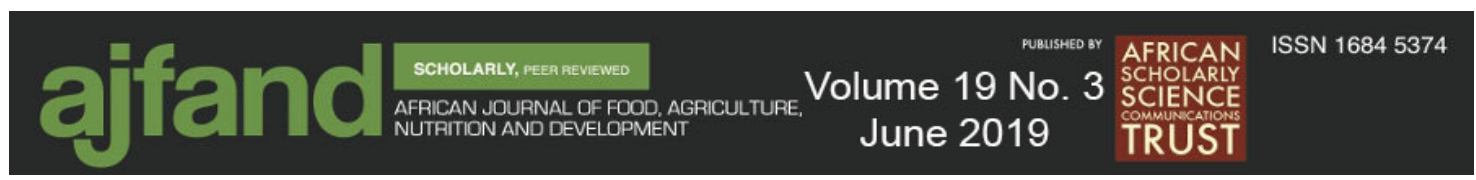

\section{RESUME}

La présente étude participe à la mécanisation de la filtration du jus de pommes d'anacarde au Bénin. Elle vise à évaluer l'efficacité technique d'un filtre-presse pour l'amélioration de la filtrabilité et de la qualité du jus de pomme de cajou. Quatre traitements ont été réalisés en filtrant deux types de jus (brut et clarifié) avec deux toiles filtrantes (en nylon et en coton) disponibles sur le marché. Le débit brut de filtration, le taux d'humidité résiduelle des gâteaux de filtration, le rendement en filtrat, la teneur en particules du filtrat et la capacité horaire du filtre-presse ont été évalués. Les caractéristiques physicochimiques, microbiologiques et sensorielles des jus issus des différents traitements ont également été déterminées. La filtration du jus clarifié avec la toile en coton a montré les meilleures performances quant au rendement moyen obtenu qui est de 56,77\%, avec une teneur en particules de 1,046 g/l de jus clarifié et une capacité de l'équipement de 212,157 $\mathrm{kg} / \mathrm{h}$. Le jus issu de ce traitement a en moyenne une teneur en sucres solubles égale à $11^{\circ}$ Brix, une teneur en vitamine $\mathrm{C}$ de $73,225 \mathrm{mg} / 100 \mathrm{~g}$ et $2,05 \mathrm{mg} / 100 \mathrm{ml}$ de tanins, avec des valeurs moyennes en magnésium et potassium respectives de 90,8 et 1174,9 mg/l. L'absence de la flore aérobie mésophile totale, des levures et moisissures, montre que tous les jus filtrés bruts ou clarifiés sont sains. Le jus clarifié et filtré avec la toile en coton a obtenu les meilleurs scores sensoriels avec sa clarté et sa couleur très acceptées. L'étude a permis de conclure que la filtration du jus de pomme de cajou clarifié à l'amidon de riz avec la toile en coton est le meilleur traitement de filtration par le filtrepresse expérimenté, parce qu'il permet de traiter plus de jus par heure et d'obtenir un jus clair de très faible teneur en particules $(1,046 \mathrm{~g} / \mathrm{l})$, avec un rendement en filtrat moyen. Le filtre-presse devra être perfectionné et mis à la disposition des unités de transformation du Bénin, pour l'amélioration de la qualité du jus de pomme de cajou.

Mots clés : pomme de cajou, jus, mécanisation, filtration, clarification, amidon de riz, filtre-presse, toiles, qualité 


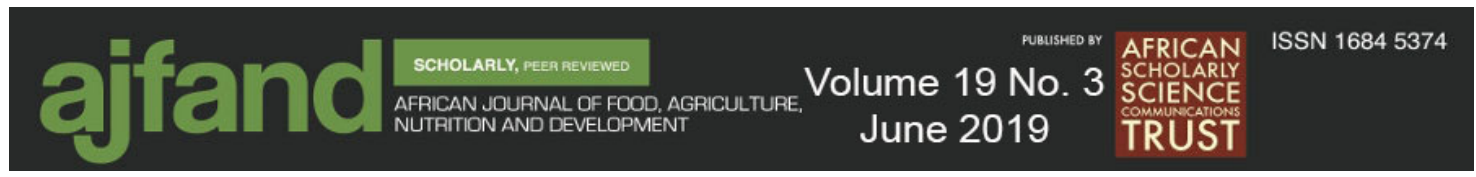

\begin{abstract}
The present study intends to contribute to the mechanization of cashew apple juice filtration process in Benin. Its aim is to evaluate the technical performances of a filter-press in order to improve cashew apple juice quality. Four treatments were tested, combining two types of juices (raw and clarified) with two types of filtration media (nylon and cotton) available on the market. Therefore, the raw flow of filtration, the residual humidity rate of filtration cakes, the filtrate yield, the filtrate particles content, the particles reduction report, the particles reduction rate, the percentage of extracted cake, and the hourly capacity of the filter-press were assessed. Physico-chemical, microbiological and sensory characteristics of juices produced from the various treatments were determined. The filtration of the clarified juice with cotton cloth showed the best performances concerning the average filtrate yield obtained which was $56.77 \%$ with particles content of $1.046 \mathrm{~g} / \mathrm{l}$ in the clarified and filtered juice and $212.157 \mathrm{~kg} /$ hour capacity of the equipment. The juice produced through that treatment had on average a Brix value of 11, a $\mathrm{pH}$ of 3.954 and a titratable acidity of $0.58 \%$. Its vitamin C content was $73.225 \mathrm{mg} / 100 \mathrm{~g}$ for a tannins content of $2.05 \mathrm{mg} / 100 \mathrm{ml}$ and respective average values in magnesium and potassium of 90.8 and $1174.9 \mathrm{mg} / \mathrm{l}$, respectively. The absence of the mesophilic bacteria, yeasts and moulds showed that raw and clarified filtered juices are healthy. The clarified juice filtered with cotton cloth obtained the best scores for its clarity and color accepted. The study concludes that the filtration of cashew apple clarified juice with the filter-press and cotton cloth, is the best treatment according to the equipment capacity, the average yield performance obtained and the lowest particles content of the juice produced. This process must be perfected and transferred to cashew apple juice producers in Benin to improve the quality of the product.
\end{abstract}

Key words: cashew apple, juice, mechanization, filtration, clarification, rice starch, filter-press, cloth, quality 


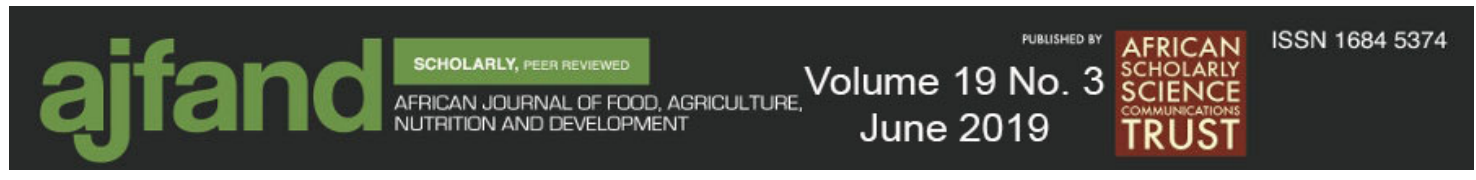

\section{INTRODUCTION}

L'anacardier (Anacardium occidentale L.) est un arbre originaire du Mexique, du Pérou, du Brésil et également des Indes occidentales. Il fournit pour l'alimentation humaine sa noix et également la pomme à laquelle la noix est attachée. La pomme est l'organe du fruit le plus important en matière de poids; près de 10 à 15 tonnes de pommes sont obtenues pour toute production d'environ une tonne de noix [1]. Malheureusement, malgré qu'elles contiennent $85 \%$ de jus riche en sucres, vitamine $\mathrm{C}$ et minéraux (magnésium et potassium), une quantité importante de pommes est abandonnée dans les champs de production, au cours de la récolte des noix [2]. Cette situation s'explique par le goût astringent du jus, dû à sa concentration en tanins de 100 à $300 \mathrm{mg} / 100 \mathrm{ml}$ [3]; la méconnaissance de sa richesse nutritionnelle et le manque de techniques appropriées pour sa transformation [4].

Au Bénin, le jus est le sous-produit le plus connu de la pomme de cajou, mais sa production nécessite une clarification, afin de le rendre plus agréable à consommer, réduisant l'astringence tout en préservant les éléments nutritifs. Différentes méthodes simples et éprouvées [5] ont donc été appliquées pour clarifier le jus de pomme de cajou dans une démarche de valorisation et la qualité ainsi que la stabilité des jus produits ont été évaluées. Mais la filtration du jus clarifié, opération manuelle réalisée avec des tissus filtrants tels que la toile mousseline, s'avère pénible, consommatrice de temps et de main d'œuvre. Le procédé de production utilisé par le groupement de femmes de Boukousséra au Nord du Bénin, montre à cet effet un système de double filtration avec une toile mousseline : la première a lieu suite au pressage des pommes et la seconde après la clarification [4]. Cependant, bien que les procédés de clarification appliqués au jus de pomme d'anacarde donnent des résultats acceptables, un dépôt tardif observable dans chaque cas persiste dans le jus filtré, conditionné et conservé en bouteilles. Parmi les diverses options technologiques qu'il est possible d'envisager pour résoudre ce problème de dépôt non apprécié des consommateurs, l'utilisation d'un filtre-presse est un procédé prometteur en raison de sa grande efficacité et sa simplicité. C'est l'un des procédés discontinus de filtration les plus utilisés dans l'industrie des boissons pour clarifier les produits intermédiaires (www.gunt.de).

Un filtre-presse adapté aux petites unités de transformation a été conçu et réalisé par le Programme de Technologies Agricoles et Alimentaires (PTAA) de l'Institut National des Recherches Agricoles du Bénin (INRAB), mais ses performances n'ont pas encore été évaluées. Aussi se pose-t-il le problème de l'identification des tissus filtrants appropriés, efficaces et disponibles localement, pour la filtration de jus de fruits.

La présente étude se propose d'évaluer l'efficacité du filtre-presse pour le traitement du jus de pomme d'anacarde et la qualité du produit. De façon spécifique il s'agit d'étudier les paramètres techniques de filtration du jus de pomme de cajou par le filtre-presse, en agissant sur le procédé et le médium de filtration; de déterminer l'effet de la filtration sur la qualité (physico-chimique, microbiologique et sensorielle) des jus brut et clarifié ; puis de déduire le tissu filtrant approprié pour la filtration du jus par le filtre-presse. 


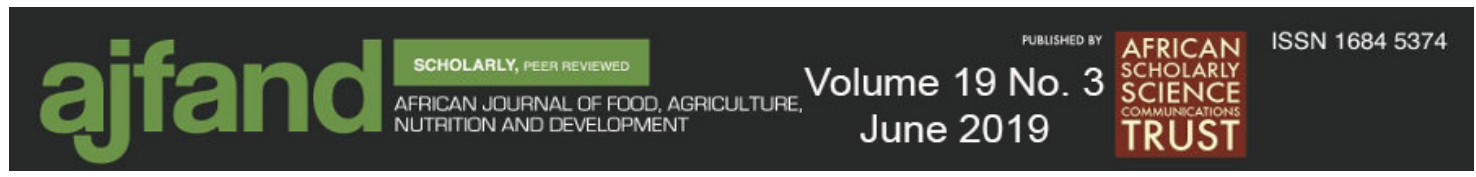

\section{METHODOLOGIE}

\section{Matière première et cadre de l'étude}

Les pommes de cajou utilisées dans cette étude ont été collectées auprès des producteurs, transportées dans des conditions de réfrigération, puis stockées dans un congélateur, à l'atelier agroalimentaire de la Faculté des Sciences Agronomiques (FSA) de l'Université d'Abomey-Calavi (UAC), qui a servi de cadre de production des jus. L'amidon de riz Nérical a été utilisé comme agent de clarification des jus. Les analyses effectuées ont ensuite été réalisées au sein de ladite Faculté, au Laboratoire de physico-chimie et de l'évaluation sensorielle des aliments et au Laboratoire des Sciences des Aliments; puis au sein de l'Institut Régional du Génie Industriel des Biotechnologies et Sciences Appliquées (IRGIB-Africa) Cotonou-Bénin, au Laboratoire de Diagnostics, d'Expertises Biologiques et Chimiques.

\section{Matériel}

Une presse Expeller, en acier inoxydable a servi au pressage des pommes après découpage. Un thermomètre à mercure a permis de contrôler la température des traitements thermiques. La filtration des jus est réalisée par le filtre-presse (photo 1), dont les composantes et données techniques sont présentées dans le tableau 1 . Il fonctionne à pression constante et comprend essentiellement deux parties. La partie électrique est constituée d'une pompe reliée au réservoir de l'équipement par un tuyau flexible, puis à la table de filtration par un tuyau métallique. La partie mécanique est composée d'une table de filtration comportant les plateaux et cadres de filtration et un plateau de serrage, tous en appui sur deux axes fixés de manière à permettre la translation des plaques. Un système de blocage des plateaux constitué d'une vis, d'un écrou et d'un contre-écrou assure le maintien en position et l'étanchéité des plateaux au cours de la filtration.

Par l'effet de la pression de la pompe, le jus est propulsé dans le filtre-presse dans un écoulement tourbillonnaire maintenant les particules solides du jus en suspension, pour les empêcher de se déposer. Le jus sous pression entre par l'orifice des plaques (trou d'admission), passe au travers des toiles filtrantes dans les chambres de filtration, pour sortir enfin par les robinets sous forme de filtrat recueilli grâce au collecteur (photo 2). Les toiles de filtration prises sur le marché local, sont en coton (toile naturelle) et en nylon (toile synthétique). Le diamètre des pores de ces toiles a été déterminé grâce à un système de criblage sur tamis superposés de mailles connues, tandis que l'épaisseur des toiles a été déterminée avec un pied à coulisse. La toile en nylon possède des pores de diamètre compris entre 0,45 et $0,63 \mathrm{~mm}$ et une épaisseur de $0,15 \mathrm{~mm}$. Le diamètre des pores de la toile en coton est inférieur à $0,1 \mathrm{~mm}$ et son épaisseur est égale à $4,2 \mathrm{~mm}$. 


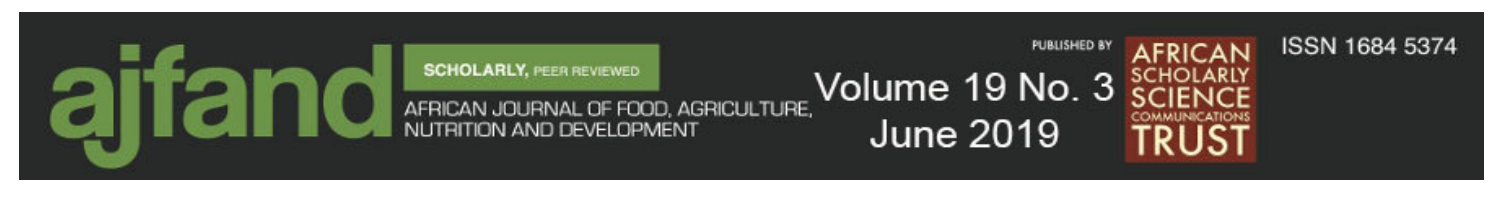

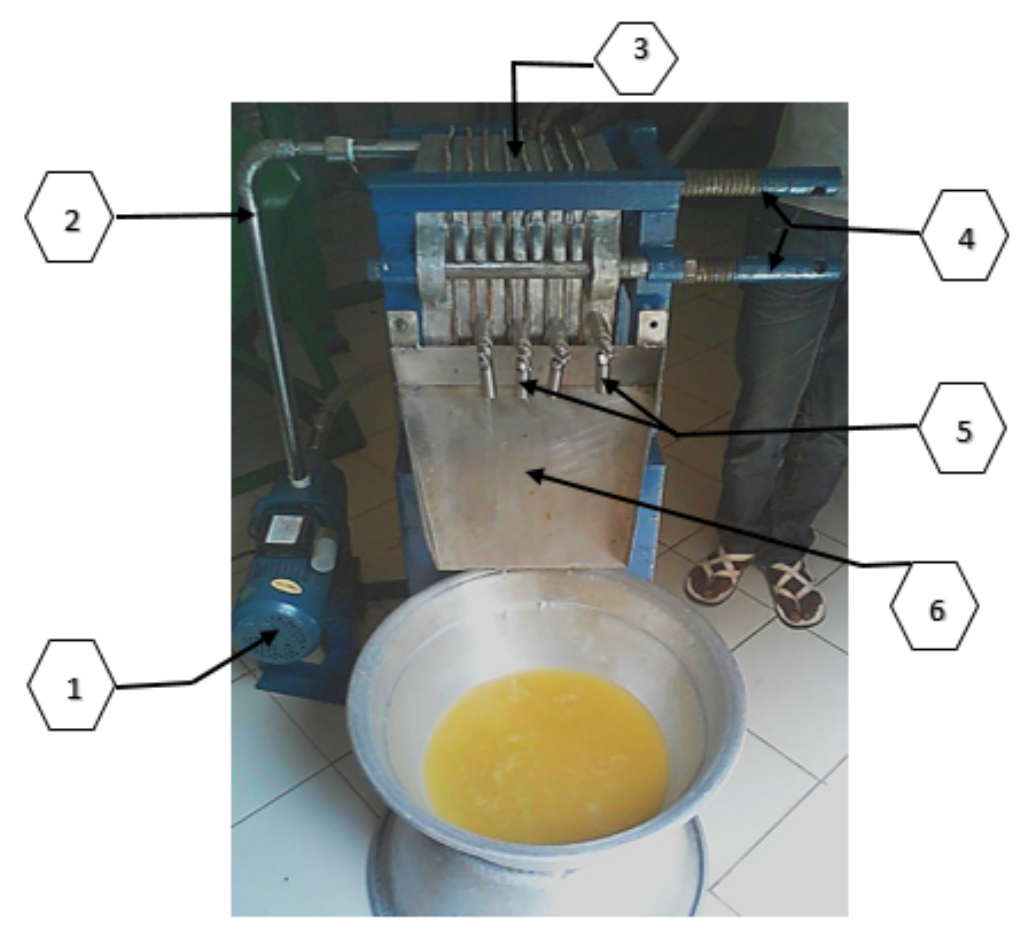

Photo 1 : Le filtre-presse

Légende :

1- pompe hydraulique

2- Tuyau métallique

3- Chambre de filtration

4- Vis de serrage

5- Robinets de sortie du filtrat

6- Collecteur de filtrat

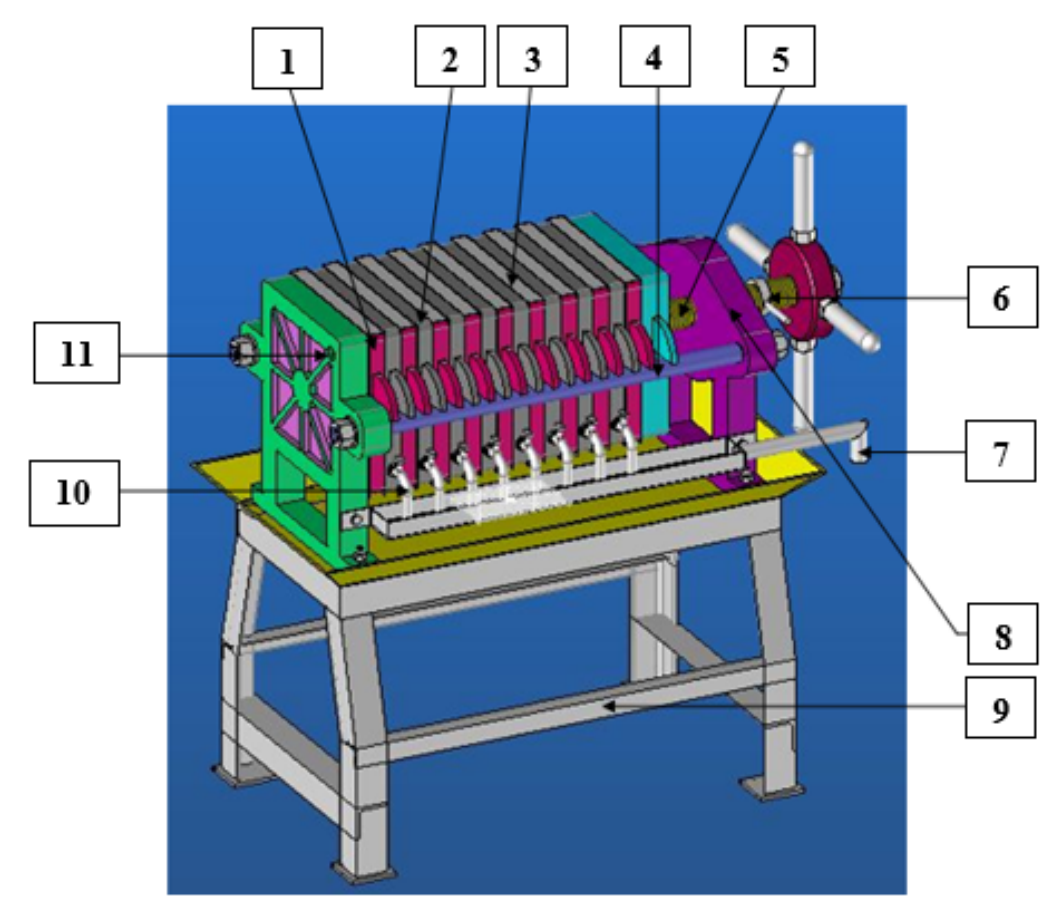

Photo 2 : Schéma de fonctionnement du filtre-presse

Légende : (1)-Cadre

(2)- Plateau de filtration

(3)- Tissu de filtration

(4)- Axe

(5)- Vis de pression

(6)- Contre-écrou
(7)- Tuyau de remplissage

(8)- Support écrou de serrage

(9)- Bâti

(10)- Robinets

(11)- Trou d'admission 


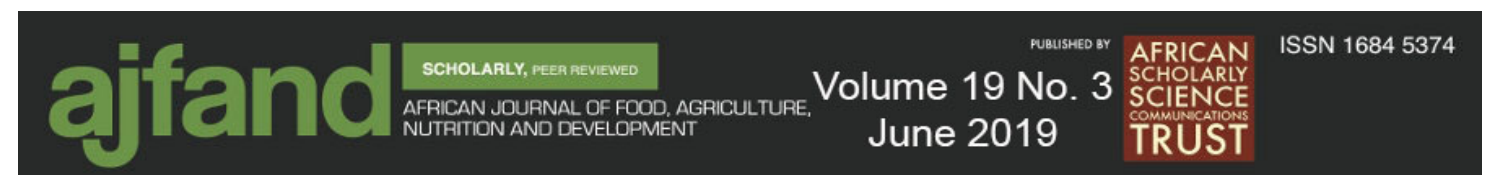

\section{Méthode de production des jus}

Les fruits ont été triés, lavés, laissés égoutter, découpés et pressés. Le jus extrait a été directement filtré ou clarifié avant d'être filtré. Vingt (20) g de poudre d'amidon de riz ont été dissous dans $100 \mathrm{ml}$ d'eau à $60^{\circ} \mathrm{C}$. Dix (10) $\mathrm{ml}$ de la solution obtenue ont ensuite été prélevés pour clarifier un litre de jus, pendant 193 minutes, pour une élimination optimale par floculation et décantation des tanins [6]. Un préchauffage du jus filtré à $60^{\circ} \mathrm{C}$ pendant environ $15 \mathrm{~min}$ a été réalisé avant la mise en bouteilles et le capsulage. Les jus ainsi conditionnés ont été pasteurisés à $80^{\circ} \mathrm{C}$ pendant $20 \mathrm{~min}$, puis refroidis.

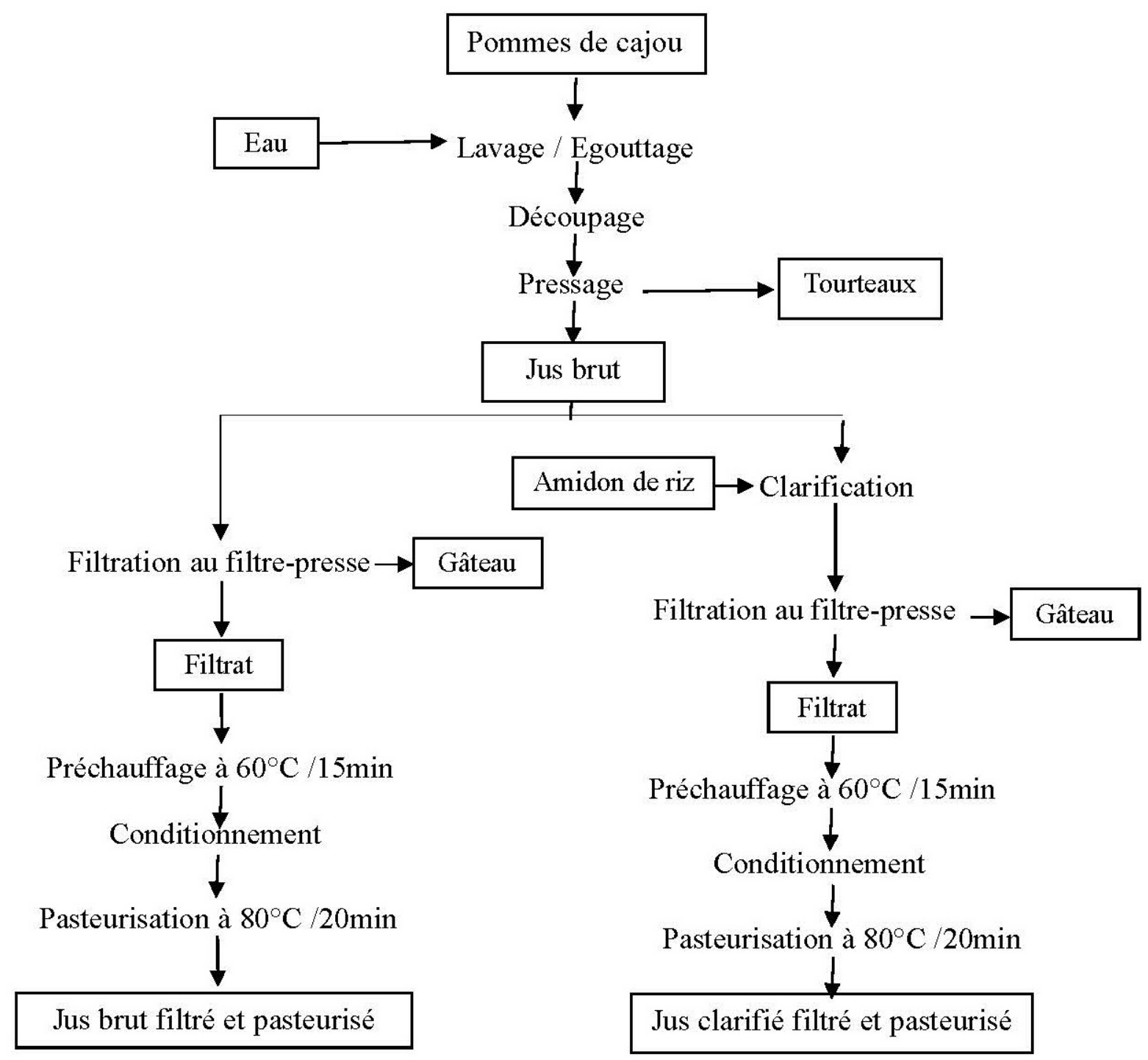

Figure 1: Diagramme technologique de production des jus de pommes de cajou filtrés au filtre-presse

\section{Traitements de filtration des jus}

Quatre (04) traitements ont été réalisés dans le cadre de cette étude :

- $\mathrm{T}_{1}$ : filtration du jus brut avec la toile en nylon aboutissant au jus de type $\mathrm{JB}_{\mathrm{n}}$;

- $\quad \mathrm{T}_{2}$ : filtration du jus brut avec la toile en coton aboutissant au jus de type $\mathrm{JB}_{\mathrm{c}}$;

- $\mathrm{T}_{3}$ : filtration du jus clarifié avec la toile en nylon aboutissant au jus de type $\mathrm{JC}_{\mathrm{n}}$; 


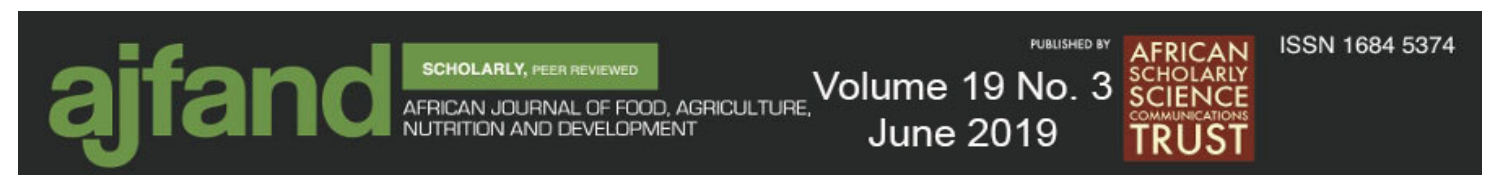

- $\mathrm{T}_{4}$ : filtration du jus clarifié avec la toile en coton aboutissant au jus de type $\mathrm{JC}_{\mathrm{c}}$. Chaque traitement a été réalisé trois fois à raison de 15 litres de jus par répétition.

\section{Détermination des paramètres d'efficacité}

L'efficacité technique du filtre-presse a été appréciée grâce à six paramètres de filtration. Le débit brut de filtration $(1 / \mathrm{h})$ qui correspond au volume de filtrat recueilli pendant une unité de temps, a été déterminé par la formule 1 :

$$
\text { Débit brut }=\frac{\text { Volume de filtrat }}{\text { Durée de filtration }}
$$

Le taux d'humidité résiduelle du gâteau de filtration sous la base humide (\%bh), correspondant au pourcentage d'humidité restée emprisonnée dans le gâteau de filtration, a été déterminé par la méthode AOAC de détermination de la teneur en eau [7].

La teneur en particules (g/l) qui exprime la quantité de matières solides (organiques ou minérales) en suspension dans le jus filtré, a été déterminée par la méthode (AFNOR T90-105), à l'aide d'un papier filtre Whatman $934 \mathrm{AH} \mathrm{N} \mathrm{N}^{\circ} 1$ qui retient les particules [8]. Les teneurs en particules des jus produits ont été comparées à celles de deux jus de pommes de cajou (Oloré et SIFRAIS) produits localement par des unités de transformation situées respectivement à Bantè et à Boukousséra au Bénin.

Le rendement en filtrat (\%), rapport entre le volume de jus filtré sortant du filtre-presse et le volume de jus y entrant, a été déterminé par la formule 2 :

$$
\text { Rendement filtrat }=\frac{\text { Volume de filtrat }}{\text { Volume jus utilisé }} \times 100
$$

La capacité horaire de l'équipement $(\mathrm{kg} / \mathrm{h})$ correspondant à la masse de jus qu'il peut filtrer par heure, a été quant à elle, déterminée par la formule 3:

$$
\text { Capacité horaire }=\frac{\text { Masse de jus utilisé }}{\text { Durée de filtration }}
$$

Enfin, la réduction en tanins qui découle de l'utilisation du filtre-presse et mesure le niveau d'élimination des facteurs antinutritionnels du jus, a été déterminée par la formule 4 :

$\%$ de réduction en tanins $=\frac{\text { Teneur en tanins dans la pomme }- \text { Teneur en tanins des jus }}{\text { Teneur en tanins dans la pomme }}$

\section{Analyse de la qualité des jus produits}

\section{Qualité physico-chimique et nutritionnelle des jus}

La qualité physico-chimique et nutritionnelle des jus de pomme de cajou produits, conditionnés en bouteilles et stockés à l'abri de la lumière, ont été appréciées à travers le $\mathrm{pH}, \mathrm{l}$ 'acidité titrable, la matière sèche soluble; les teneurs en tanins, minéraux et en vitamine $\mathrm{C}$. Le $\mathrm{pH}$ a été déterminé sur chaque type de jus au moyen d'un $\mathrm{pH}$-mètre, préalablement calibré avec des solutions tampon de $\mathrm{pH}$ 4,0 et 7,0. L'acidité titrable en pourcentage d'acide malique, a été déterminée par titration avec du $\mathrm{NaOH}(0,1 \mathrm{~N})$ en présence de phénolphtaléine selon la méthode ISO750:1998. La matière sèche soluble ( $\left.{ }^{\circ} \mathrm{Brix}\right)$ est déterminée à $20^{\circ} \mathrm{C}$ au moyen d'un 


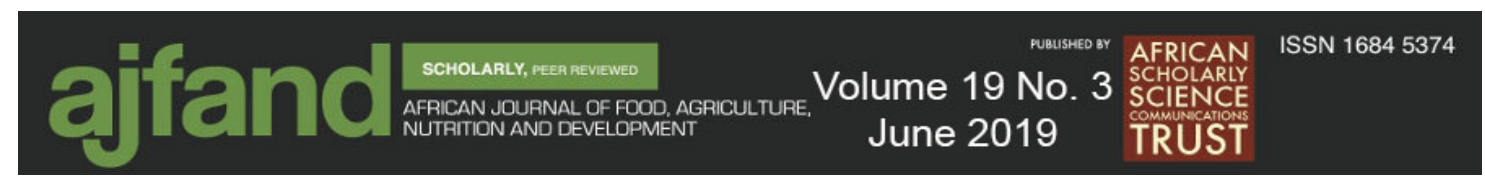

réfractomètre portatif à affichage numérique. La couleur des jus est évaluée grâce au chromamètre CR-480 qui fournit les paramètres $\mathrm{L}^{*}, \mathrm{a}^{*}, \mathrm{~b}^{*}$ servant au calcul de l'indice de brunissement (IB) des jus suivant les formules 5.1 et 5.2 :

$$
\begin{gathered}
I B=\frac{(x-0,31)}{0,17} \\
\text { Avec : } x=\frac{(\mathrm{a} *+1,75 \mathrm{~L} *)}{5,645 \mathrm{~L} *+(\mathrm{a} *-3,012 \mathrm{~b} *)}
\end{gathered}
$$

Les tanins sont déterminés par la méthode à la tyrosine (Hach-Method 8193) en présence d'un réactif (TanniVer3), qui produit dans l'échantillon une coloration bleue proportionnelle à la quantité de composés tanniques. La lecture se fait en $\mathrm{mg} / \mathrm{l}$ d'acide tannique, grâce à un colorimètre portatif $\mathrm{DR} / 890$ de marque $\mathrm{HACH}$. Le potassium et le magnésium sont déterminés par Spectrométrie d'Absorption Atomique (SAA 220) à l'air-acétylène, révélés par une lampe UV avec les longueurs d'ondes respectives de 285,2 et 769,9 nm. La teneur en vitamine $C$ est déterminée par titration au 2,6-dichlorophénolindophénol (2,6-DCPIP) à 1,3.10-3 $\mathrm{mol} / \mathrm{L}$.

\section{Qualité microbiologique des jus}

Elle a été appréciée par détermination de la flore aérobie mésophile sur milieu Plate Count Agar (PCA), dénombrée selon la norme ISO 4833: 2003 et des levures et moisissures déterminées sur gélose Sabouraud (SDA OXOID CM0041) au chloramphénicol, selon la norme NF ISO 7954.

\section{Qualité sensorielle des jus}

Un panel de 30 dégustateurs amateurs a servi à l'évaluation sensorielle des jus au moyen de tests axés sur les produits et les consommateurs. Les critères d'appréciation concernant la clarté et la couleur ont été évalués, de même que les goûts astringent, sucré et acide. Les dégustateurs ont identifié par la suite le jus dont ils ont le plus aimé chacun des critères évalués.

\section{Traitement des données et analyses statistiques}

Les données générées par l'expérimentation sont traitées par le tableur Excel (Microsoft office) pour les calculs. L'analyse de la variance s'est faite par le test paramétrique ANOVA et le test non paramétrique Kruskal Wallis, en utilisant le logiciel Minitab 14. La méthode de Tukey est utilisée pour obtenir les différences deux à deux entre les moyennes des niveaux des facteurs (procédé et toiles filtrantes) au cas où l'un au moins influence la variable testée.

\section{RESULTATS ET DISCUSSION}

\section{Performances techniques du filtre-presse}

L'analyse du tableau 2 montre que la variation du débit brut de filtration ne dépend que de la toile de filtration. Les valeurs moyennes obtenues sont élevées pour les traitements de filtration par la toile en coton, soit 135,218 1/h et $120,70 \mathrm{l} / \mathrm{h}$ respectivement pour $\mathrm{T}_{2}$ et $\mathrm{T}_{4}$. Aussi, le taux d'humidité résiduelle des gâteaux de filtration varie significativement sous l'influence du procédé de clarification. Sa valeur est plus faible pour les jus clarifiés et filtrés. Le traitement $\mathrm{T}_{4}$ permet d'obtenir le plus faible taux d'humidité résiduelle du gâteau de filtration qui est de $82,07 \%$ bh (base humide). 


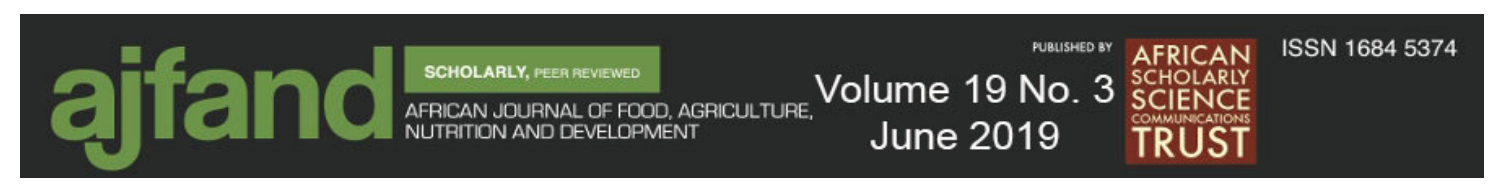

La figure 2 illustre les différentes teneurs en particules des jus filtrés issus des quatre traitements $T_{1}, T_{2}, T_{3}$ et $T_{4}$. Il existe entre ces teneurs, une différence significative au seuil de $5 \%$ relative au type de toile de filtration et à la clarification.

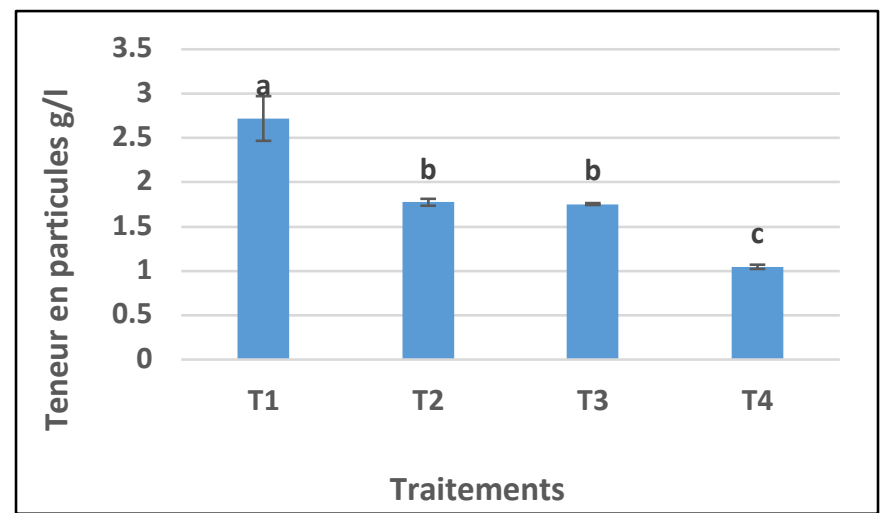

Figure 2: Teneurs en particules des jus filtrés

Les jus bruts filtrés ont des teneurs en particules moyennes de 1,754 et 2,719 g/1 respectivement pour les traitements $T_{2}$ et $T_{1}$; tandis que celles des jus clarifiés et filtrés sont de 1,046 et 1,774 $\mathrm{g} / \mathrm{l}$ respectivement pour les traitements $\mathrm{T}_{4}$ et $\mathrm{T}_{3}$.

Le rendement moyen en filtrat fourni par le filtre-presse pour les quatre traitements varie entre 56,77 et $69,46 \%$ aussi bien en fonction de la toile de filtration que du procédé (figure 3). Les traitements révèlent une influence du procédé et de la toile de filtration sur la capacité horaire $\mathrm{du}$ filtre-presse. En effet, les valeurs moyennes obtenues (figure 4) sont élevées pour les traitements à la toile en coton (respectivement $189,786 \mathrm{~kg} / \mathrm{h}$ et $212,157 \mathrm{~kg} / \mathrm{h}$ pour $\mathrm{T}_{2}$ et $\mathrm{T}_{4}$ ), comparativement aux traitements à la toile en nylon dont les valeurs sont faibles, soient 150,849 $\mathrm{kg} / \mathrm{h}$ pour $\mathrm{T}_{1}$ et $138,824 \mathrm{~kg} / \mathrm{h}$ pour $\mathrm{T}_{3}$.

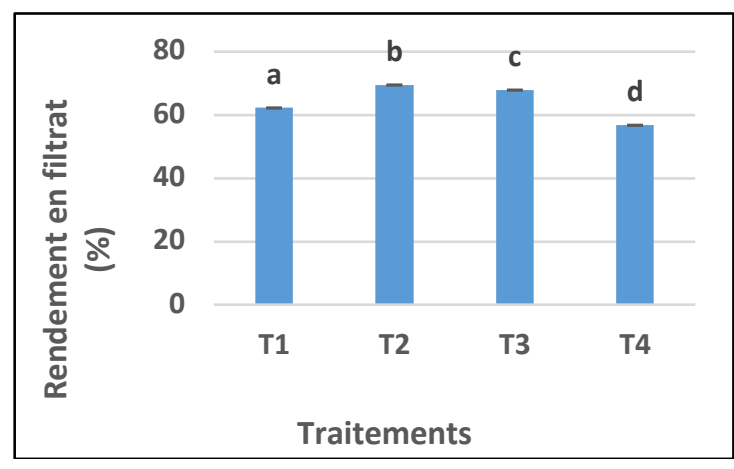

Figure 3: Rendement en filtrat du filtre-presse

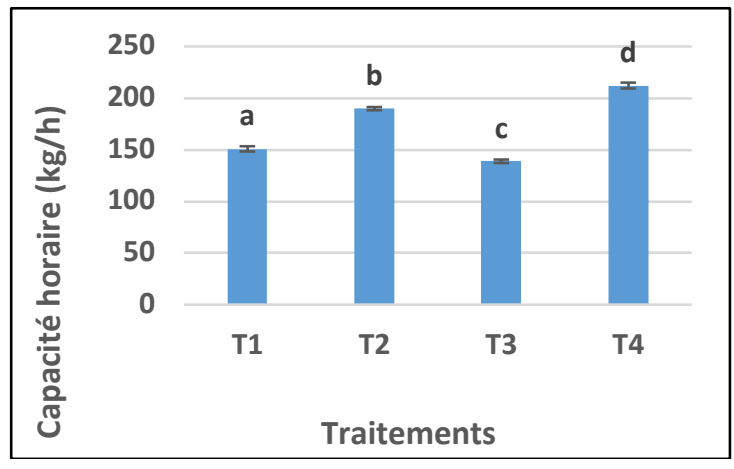

Figure 4: Capacité horaire du filtre-presse

Des analyses ci-dessus, associées à une analyse en composante principale (ACP) tenant compte du producteur dont le souci est de pouvoir produire en grande quantité (capacité horaire élevée) et avec un bon rendement, du jus " clarifié » de pommes de cajou dépourvu de particules, il ressort que le traitement $\mathrm{T}_{4}$ est celui qui permet au filtre-presse d'afficher les meilleures performances techniques (figure 5). Ce procédé offre la possibilité de traiter plus de jus par heure $(212,157 \mathrm{~kg} / \mathrm{h})$ et d'obtenir un jus clair de très faible teneur en particules $(1,046 \mathrm{~g} / \mathrm{l})$. 

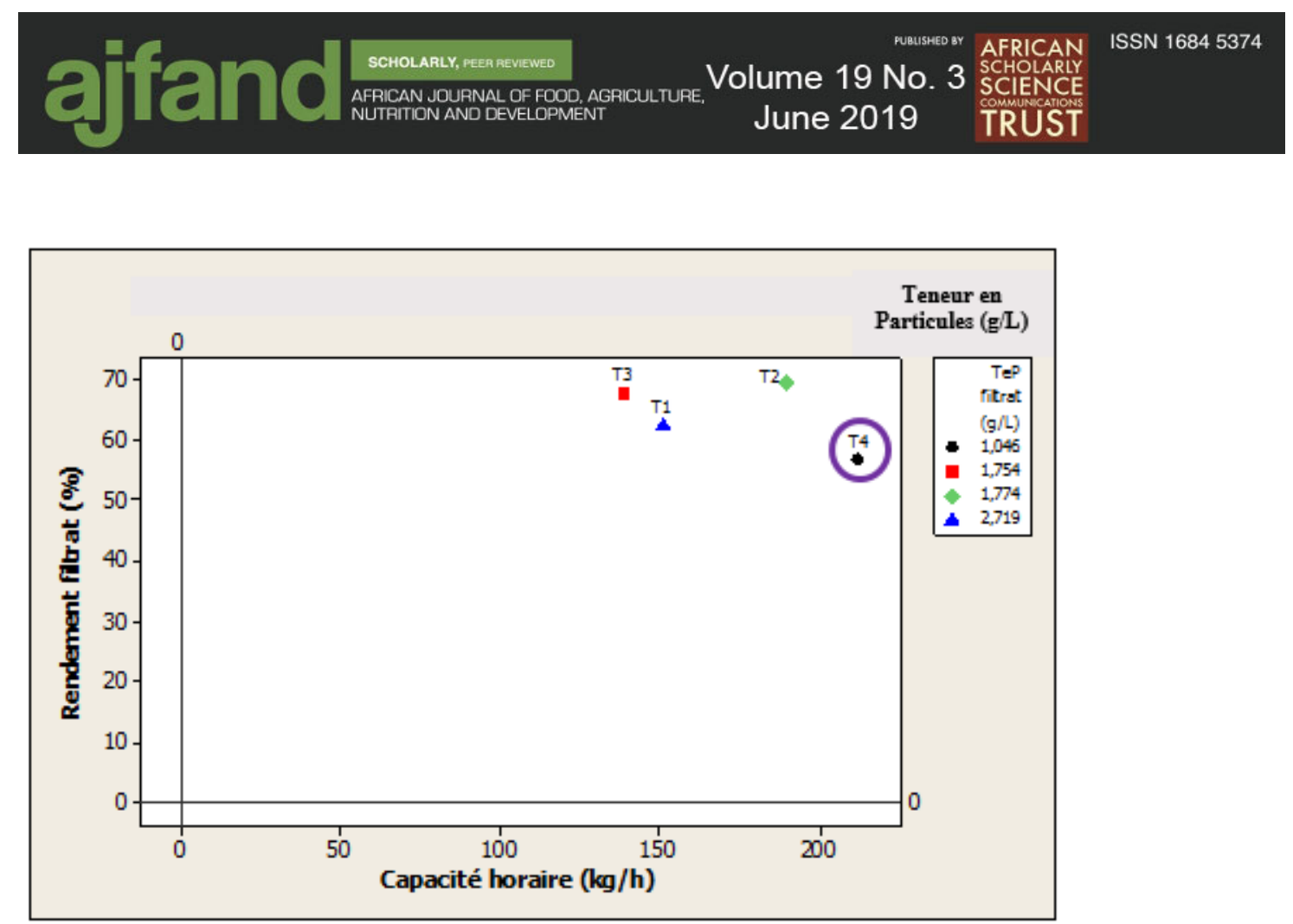

Figure 5: Regroupement des traitements en fonction de trois paramètres

L'utilisation du filtre-presse a révélé des performances techniques appréciables en ce qui concerne la réduction des particules en suspension liées à la sédimentation tardive, le rendement en filtrat et la réduction du taux initial en tanins.

Par rapport au taux de particules des jus, la clarification a certes prouvé son efficacité, puisque les teneurs en particules des jus bruts sont supérieures à celles des jus clarifiés. Mais l'effet de la filtration est encore plus perceptible et dépend de la toile utilisée. Les traitements à la toile en nylon réduisent moins les particules que les traitements à la toile en coton. La floculation des particules en suspension dans les jus clarifiés et la maille de la toile en coton peuvent expliquer ce phénomène. En effet, pour arrêter les petites particules, il convient de procéder à la floculation afin d'obtenir des particules plus grosses et donc plus faciles à filtrer (www.eduscol.education.fr). Le diamètre des pores de la toile en coton étant le plus faible, cette dernière a donc retenu plus de particules en suspension que la toile en nylon. Par conséquent, le traitement $\mathrm{T}_{4}$ de filtration de jus clarifié et filtré avec la toile en coton, révèle une réduction d'environ 20 fois la teneur en particules du jus avant la filtration $(21,323 \mathrm{~g} / 1)$, comparativement au traitement $T_{3}$ de filtration de jus clarifié et filtré avec la toile nylon, qui révèle une réduction d'environ 12 fois. Les teneurs en particules des jus clarifiés et filtrés $\left(\mathrm{JC}_{\mathrm{n}}\right.$ et $\left.\mathrm{JC}_{\mathrm{c}}\right)$ sont inférieures à celles des jus «Oloré» de Bantè et «SIFRAIS» de Boukousséra (respectivement 4,651 et 2,501 $\mathrm{g} / \mathrm{l})$. En effet, les échantillons «Oloré» clarifié avec l'amidon de manioc et «SIFRAIS» clarifié au gruau de riz, sont issus d'opération manuelle de filtration, avec une toile mousseline, avant et après la clarification [9]. Les valeurs de rendement en filtrat obtenues pour les traitements des jus clarifiés $\mathrm{T}_{4}(56,77 \%)$ et $\mathrm{T}_{3}(67,86 \%)$ sont inférieures aux valeurs moyennes $(83,47$ et $89,17 \%$ ) obtenues pour la production des jus clarifiés à l'amidon de manioc et au gruau de riz puis filtrés manuellement avec une toile mousseline [9]. Notons que dans la présente étude, le jus de pomme de cajou n'est pas filtré avant la clarification et le dépôt de clarification n'est pas évacué avant la filtration par filtre-presse. Ce dépôt forme le gâteau de filtration qui joue ainsi le rôle d'un second médium de filtration. 


\section{Effet des traitements sur les caractéristiques physico-chimiques des jus}

L'analyse du tableau 3 présentant le degré Brix, le $\mathrm{pH}$ et l'acidité titrable des jus de pomme de cajou, ressort que les jus clarifiés ont une teneur en matières sèches solubles supérieure à celles des jus bruts. Les valeurs de Brix des jus bruts $\left(\mathrm{JB}_{\mathrm{n}}\right.$ et $\left.\mathrm{JB}_{\mathrm{c}}\right)$ sont inférieures aux résultats obtenus au Nigéria [10] et en Côte d'ivoire [11] qui sont respectivement 10,2 et 10,9 ${ }^{\circ}$ Brix. Les jus clarifiés $\left(\mathrm{JC}_{\mathrm{n}}\right.$ et $\left.\mathrm{JC}_{\mathrm{c}}\right)$ ont des degrés Brix proches de 11, valeur obtenue pour le jus de pomme de cajou clarifié au gruau de riz [9]. Les teneurs en sucres solubles des jus $\mathrm{JC}_{\mathrm{n}}$ et $\mathrm{JC}_{\mathrm{c}}$ sont plus élevées que $10^{\circ}$ Brix, valeur minimale prévue pour le jus de pomme de cajou par la norme brésilienne [12]. La significativité des différences au niveau du $\mathrm{pH}$ des jus ne dépend ni de la toile de filtration, ni du procédé, mais pourrait être liée aux pommes elles-mêmes. En effet, la nature et la composition du sol, les pratiques culturales et les conditions climatiques [13], de même que les couleurs et formes des pommes de cajou [11] et le niveau de maturité des fruits à la récolte [14], affectent la composition physico-chimique des pommes de cajou et pourraient être à l'origine de ces différents résultats. Mais l'acidité titrable des jus ne révèle pas de différence significative. Sachant que le $\mathrm{pH}$ reflète la nature acide des produits, la différence significative des valeurs de $\mathrm{pH}$ observées dans cette étude semble indiquer que le jus $\mathrm{JC}_{\mathrm{n}}$ aurait dans sa composition chimique, une proportion d'acides faibles plus élevée, contrairement aux jus $\mathrm{JB}_{\mathrm{n}}, \mathrm{JB}_{\mathrm{c}}$ et $\mathrm{JC}_{\mathrm{c}}$. Les $\mathrm{pH}$ des jus $\mathrm{JB}_{\mathrm{n}}$ et $\mathrm{JB}_{\mathrm{c}}$ sont inférieurs aux $\mathrm{pH}$ (respectivement 4,1 et 4,3$4,5)$ obtenus au Nigéria et en Côte d'ivoire pour le jus brut de pomme cajou [10, 11].

La couleur des jus $\mathrm{JB}_{n}$ et $\mathrm{JC}_{n}$ est plus claire en comparaison aux jus $\mathrm{JB}_{\mathrm{c}}$ et $\mathrm{JC}_{\mathrm{c}}$ comme l'indiquent les indices de brunissement dont les valeurs sont les plus élevées, soit 0,184 et 0,17 respectivement pour $\mathrm{JB}_{\mathrm{c}}$ et $\mathrm{JC}_{\mathrm{c}}$, que pour $\mathrm{JB}_{\mathrm{n}}$ et $\mathrm{JC}_{\mathrm{n}}(0,134$ et 0,12 respectivement).

\section{Qualité nutritionnelle des jus filtrés au filtre-presse}

Les teneurs en minéraux des jus sont indiquées dans le tableau 4. Il ressort que le jus clarifié et filtré avec la toile en coton $\left(\mathrm{JC}_{\mathrm{c}}\right)$, présente des teneurs en magnésium $(\mathrm{Mg})$ et potassium $(\mathrm{K})$ de $90,8 \mathrm{mg} / 1$ et $1174,9 \mathrm{mg} / \mathrm{l}$ respectivement. Ces valeurs sont supérieures à celles du jus brut filtré avec la toile en coton $\left(\mathrm{JB}_{\mathrm{c}}\right)$. Par contre, le jus clarifié et filtré à la toile nylon $\left(\mathrm{JC}_{\mathrm{n}}\right)$ a une teneur en $\mathrm{Mg}(78,4 \mathrm{mg} / \mathrm{l})$ inférieure à celle du jus brut $\mathrm{JB}_{\mathrm{n}}(85,7 \mathrm{mg} / \mathrm{l})$, bien que sa teneur en $\mathrm{K}$ soit élevée. En ce qui concerne la vitamine $\mathrm{C}$ (tableau 5), les jus bruts présentent des valeurs (respectivement 162,635 et $151,455 \mathrm{mg} / 100 \mathrm{~g}$ pour $\mathrm{JB}_{\mathrm{n}}$ et $\mathrm{JB}_{\mathrm{c}}$ ) supérieures à celles des jus clarifiés qui sont respectivement de 58,74 et $73,225 \mathrm{mg} / 100 \mathrm{~g}$ pour $\mathrm{JC}_{\mathrm{n}}$ et $\mathrm{JC}_{\mathrm{c}}$. La différence observée pour ces teneurs en vitamine $\mathrm{C}$ des jus pourrait s'expliquer de plusieurs manières. En effet, l'exsudation des aliments congelés pendant la décongélation constitue une perte en substances nutritives, surtout les vitamines en particulier [15]. Or les pommes ont été congelées avant leur utilisation pour l'expérimentation dans cette étude. La clarification réduit également la teneur en vitamine $C$ dans les jus clarifiés [16]. A cela s'ajoute, l'oxydation rapide de la vitamine lors de la pasteurisation appliquée aux différents jus après la clarification [17]. Cette réaction nettement mise en évidence à partir de $60^{\circ} \mathrm{C}$, semble être le principal facteur responsable du brunissement du jus [18]. Mais malgré les pertes, les jus clarifiés $\mathrm{JC}_{n}$ et $\mathrm{JC}_{\mathrm{c}}$ contiennent plus de vitamine $\mathrm{C}$ que la dose journalière recommandée pour un adulte qui est de $45 \mathrm{mg}$ [19]. Ils contiennent toutefois moins de magnésium et potassium que les apports recommandés dont les valeurs sont respectivement $1600 \mathrm{mg}$ et $300 \mathrm{mg}$ par jour [20]. 


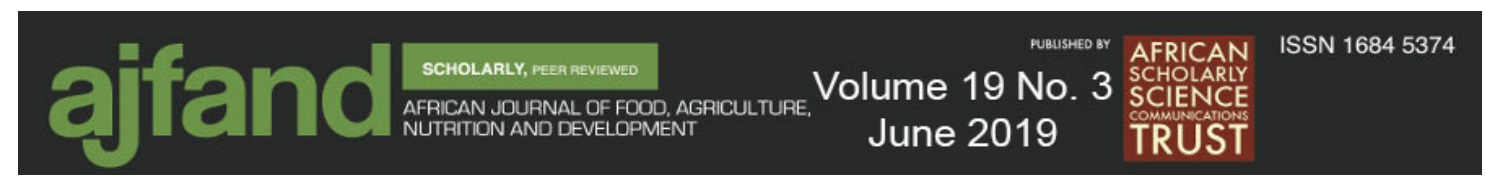

\section{Impacts de la filtration sur les paramètres antinutritionnels}

Le tableau 5 présente les teneurs en tanins des jus. Les teneurs en tanins des jus clarifiés $\left(\mathrm{JC}_{\mathrm{n}}\right.$ et $\mathrm{JC}_{\mathrm{c}}$ ) sont faibles, respectivement 2,81 et $2,05 \mathrm{mg}$ pour $100 \mathrm{ml}$ de jus ; alors que celles des jus bruts varient de $6,6 \mathrm{mg} / 100 \mathrm{ml}$ pour $\mathrm{JB}_{\mathrm{c}}$ à $13,745 \mathrm{mg} / 100 \mathrm{ml}$ pour $\mathrm{JB}_{\mathrm{n}}$. Les teneurs en tanins des jus bruts sont inférieures à celles obtenues par certains auteurs, dont les valeurs varient entre 100 et $300 \mathrm{mg} / 100 \mathrm{ml}[21,22,23]$. Ces différences peuvent être associées aux variations génétiques et climatiques, qui influencent significativement la teneur en tanins de la pomme de cajou selon certains auteurs $[23,24]$. En ce qui concerne les jus clarifiés $\mathrm{JC}_{\mathrm{n}}$ et $\mathrm{JC}_{\mathrm{c}}$, les teneurs en tanins sont inférieures aux valeurs ( 5 à $8 \mathrm{mg} / 100 \mathrm{ml}$ ) obtenues par la microfiltration tangentielle avec un prétraitement enzymatique des jus de pomme de cajou [21]. La filtration par filtre-presse réalisée dans la présente étude, a réduit de 86 à $98 \%$ pour les jus bruts et 97 à $99 \%$ pour les jus clarifiés, la teneur en tanins des pommes de cajou [3]. Le traitement se révèle donc efficace en comparaison au traitement réalisant la clarification à l'amidon de riz par décantation suivie du siphonage par trop-plein et de la filtration du surnageant clair à l'aide d'une toile mousseline, qui montre une réduction de 42,14\% des tanins [6]. Il est également efficace par rapport aux travaux de clarification du jus avec le « sago ", montrant une réduction de $42,85 \%$ des tanins [25].

\section{Qualité sanitaire des jus filtrés}

Il y a une absence de la flore aérobie mésophile totale (FAMT), des levures et moisissures recherchées dans les jus aussi bien bruts que clarifiés, quel que soit la toile de filtration utilisée (nylon ou coton). Ces résultats démontrent que les conditions du milieu sont défavorables au développement des microorganismes d'altération. Cela pourrait s'expliquer par le double traitement thermique [9] $\left(60^{\circ} \mathrm{C}\right.$ pendant $15 \mathrm{~min}$ et $80^{\circ} \mathrm{C}$ pendant $\left.20 \mathrm{~min}\right)$, mais essentiellement la pasteurisation à $80^{\circ} \mathrm{C}$. Le $\mathrm{pH}$ bas des jus empêche également que certains microorganismes (tels que les staphylocoques et Clostridium botulinum) se développent [26].

\section{Qualité sensorielle des jus filtrés}

La clarté du jus $\mathrm{JC}_{\mathrm{c}}$ a reçu le meilleur score d'appréciation, de même que sa couleur. Les dégustateurs sont satisfaits de l'élimination du dépôt dans le jus clarifié $\mathrm{JC}_{\mathrm{c}}$ dont la teneur en particules est la plus faible $(1,046 \mathrm{~g} / 1)$. Sa couleur ni trop pâle ni trop foncée est jugée fortement agréable. L'astringence des jus clarifiés n'est plus remarquée contrairement aux jus bruts pour lesquels une astringence légère est distinguée. Cela semble en concordance avec les faibles teneurs en tanins de 2,81 et 2,05 mg pour $100 \mathrm{ml}$ pour les jus clarifiés $\mathrm{JC}_{\mathrm{n}}$ et $\mathrm{JC}_{\mathrm{c}}$ respectivement. Les jus bruts sont appréciés comme étant moins sucrés que les jus clarifiés. Ceci est probablement dû à l'astringence du jus brut qui semble voiler la perception du sucré ; l'astringence du jus clarifié n'étant plus perceptible, le goût sucré se révèle donc plus. Aussi, Parmi tous les jus, $\mathrm{JB}_{\mathrm{n}}$ est apprécié comme étant le plus acide. L'acidité faible perçue pour $\mathrm{JC}_{\mathrm{n}}$ s'accorde avec les valeurs de $\mathrm{pH}$ des jus montrant pour $\mathrm{JC}_{\mathrm{n}}$ un $\mathrm{pH}$ de 4,155 élevé par rapport aux $\mathrm{pH}$ des autres jus qui varient entre 3,943 et 3,988. L'odeur des pommes de cajou est cependant perçue dans chaque jus, mais elle est plus intense pour les jus bruts que pour les jus clarifiés. Le jus clarifié et filtré à la toile en coton $\left(\mathrm{JC}_{\mathrm{c}}\right)$ a été préféré pour tous les critères d'évaluation. Il est suivi du jus clarifié et filtré et à la toile en nylon $\mathrm{JC}_{\mathrm{n}}$, puis des jus bruts filtrés à la toile en coton $\left(\mathrm{JB}_{\mathrm{c}}\right)$ et à la toile en nylon $\left(\mathrm{JB}_{\mathrm{n}}\right)$ respectivement. 


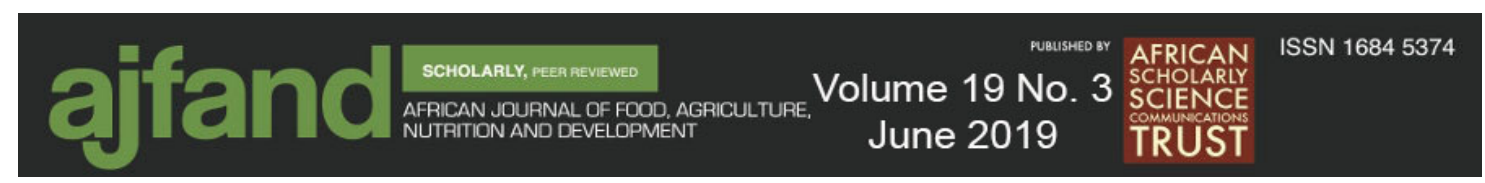

\section{CONCLUSION}

La présente étude rend compte des effets de la filtration par filtre-presse sur la qualité et l'acceptabilité du jus de pomme de cajou. Elle répond aux attentes des producteurs de jus de pommes de cajou, en proposant le traitement de filtration du jus clarifié de pomme de cajou avec la toile en coton, comme étant la formule de production de jus de pomme de cajou la plus intéressante. La toile en coton s'est révélée la meilleure toile filtrante. En perspective, nous envisageons d'expérimenter le filtre-presse dans les conditions réelles des unités de production de jus de pomme de cajou au Bénin, en utilisant la toile en coton et améliorer le procédé pour le faire adopter par ces unités. A moyen termes, il est envisagé la diffusion de cette technologie auprès des petites et moyennes unités de transformation de la pomme cajou en jus au Bénin.

\section{REMERCIEMENTS}

La présente recherche a été réalisée dans le cadre de la mécanisation de la filtration des jus de pommes d'anacarde au Bénin. Les auteurs sont reconnaissants et expriment leur gratitude au Programme de Technologies Agricoles et Alimentaires (PTAA) pour la mise au point du filtrepresse utilisé dans le cadre des travaux. 


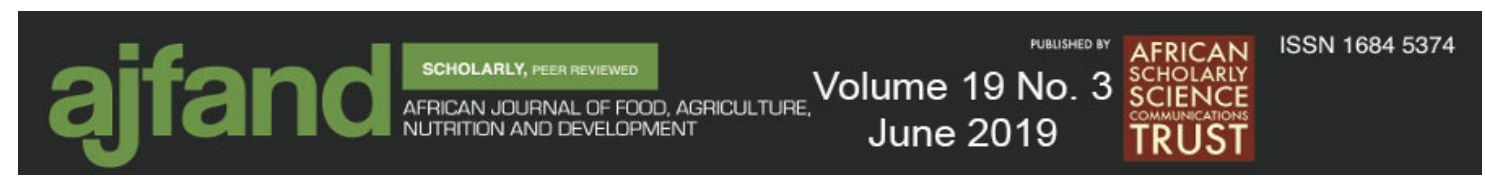

Tableau 1: Composantes et données techniques du filtre-presse

\section{Caractéristiques de l'équipement}

\begin{tabular}{lc}
\hline Nombre de plateaux de filtration par robinet & 04 \\
Dimensions des plateaux $(\mathrm{mm} \times \mathrm{mm})$ & $230 \times 230$ \\
Nombre de cadres & 05 \\
Nombre de toiles & 08 \\
Capacité du réservoir $(\mathrm{l})$ & 20 \\
Fréquence du moteur $(\mathrm{Hz})$ & 50 \\
Nombre de phases du moteur & 01 \\
Vitesse de rotation du moteur (r.p.m) & 2850 \\
Débit de la pompe $(1 / \mathrm{min})$ & 6 à 60 \\
\hline
\end{tabular}

Tableau 2: Valeurs du débit brut et du taux d'humidité résiduelle des gâteaux de filtration

\begin{tabular}{ccc}
\hline Traitements & Débit brut de filtration $(\mathbf{l} / \mathbf{h})$ & Humidité résiduelle du gâteau $(\% \mathbf{b h})$ \\
\hline $\mathbf{T}_{\mathbf{1}}$ & $98,548 \pm 1,673^{\mathrm{a}}$ & $84,640 \pm 0,829^{\mathrm{a}}$ \\
$\mathbf{T}_{\mathbf{2}}$ & $135,218 \pm 1,234^{\mathrm{b}}$ & $83,116 \pm 0,091^{\mathrm{a}}$ \\
$\mathbf{T}_{\mathbf{3}}$ & $97,346 \pm 1,062^{\mathrm{a}}$ & $82,274 \pm 0,308^{\mathrm{b}}$ \\
$\mathbf{T}_{\mathbf{4}}$ & $120,703 \pm 1,663^{\mathrm{b}}$ & $82,073 \pm 0,060^{\mathrm{b}}$ \\
\hline
\end{tabular}

Dans une même colonne, les moyennes avec les mêmes lettres ne sont pas significativement différentes à 5\% T1: filtration du jus brut avec la toile en nylon; $\mathbf{T}_{2}$ : filtration du jus brut avec la toile en coton; $\mathbf{T}_{3}$ : filtration du jus clarifié avec la toile en nylon; T4: filtration du jus clarifié avec la toile en coton, \%bh: Pourcentage en base humide

Tableau 3: Degré Brix, pH et acidité titrable des jus de pommes de cajou

\begin{tabular}{cccc}
\hline Jus & Degré Brix & $\mathbf{p H}$ & Acidité titrable (\%) \\
\hline $\mathbf{J B}_{\mathbf{n}}$ & $9,967 \pm 0,058^{\mathrm{a}}$ & $3,943 \pm 0,030^{\mathrm{a}}$ & $0,673 \pm 0,010^{\mathrm{a}}$ \\
$\mathbf{J B}_{\mathbf{c}}$ & $10,6 \pm 0^{\mathrm{b}}$ & $3,988 \pm 0,007^{\mathrm{bc}}$ & $0,564 \pm 0,005^{\mathrm{a}}$ \\
$\mathbf{J C}_{\mathbf{n}}$ & $10,267 \pm 0,058^{\mathrm{c}}$ & $4,155 \pm 0,008^{\mathrm{b}}$ & $0,530 \pm 0,010^{\mathrm{a}}$ \\
$\mathbf{J C}_{\mathbf{c}}$ & $11 \pm 0^{\mathrm{d}}$ & $3,954 \pm 0,006^{\mathrm{c}}$ & $0,579 \pm 0,011^{\mathrm{a}}$
\end{tabular}

Dans une même colonne, les moyennes avec les mêmes lettres ne sont pas significativement différentes à 5\% 


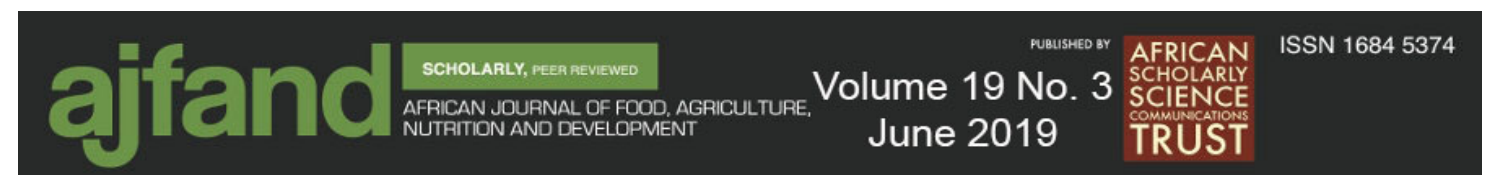

Tableau 4: Teneurs en minéraux des jus de pommes de cajou

\begin{tabular}{ccc}
\hline Jus & Magnésium (mg/l) & Potassium (mg/l) \\
\hline $\mathbf{J B}_{\mathbf{n}}$ & $85,7 \pm 1,556^{\mathrm{a}}$ & $1126 \pm 0,566^{\mathrm{a}}$ \\
$\mathbf{J B}_{\mathbf{c}}$ & $69 \pm 0,566^{\mathrm{a}}$ & $1108,05 \pm 0,354^{\mathrm{a}}$ \\
$\mathbf{J C}_{\mathbf{n}}$ & $78,4 \pm 0,424^{\mathrm{a}}$ & $1144,7 \pm 1,556^{\mathrm{b}}$ \\
$\mathbf{J C}_{\mathbf{c}}$ & $90,8 \pm 0,849^{\mathrm{a}}$ & $1174,9 \pm 0,283^{\mathrm{b}}$
\end{tabular}

Dans une même colonne, les moyennes avec les mêmes lettres ne sont pas significativement différentes à $5 \%$

Tableau 5: Teneurs en vitamine $C$ et en tanins des jus de pommes de cajou

\begin{tabular}{cccc}
\hline Jus & $\begin{array}{c}\text { Tanins } \\
(\mathbf{m g} / \mathbf{1 0 0 m})\end{array}$ & $\begin{array}{c}\text { \% de réduction } \\
\text { en tanins }\end{array}$ & $\begin{array}{c}\text { Vitamine C } \\
(\mathbf{m g} / \mathbf{1 0 0 g})\end{array}$ \\
\hline $\mathbf{J B}_{\mathbf{n}}$ & $13,745 \pm 0,926^{\mathrm{a}}$ & $86-95$ & $162,635 \pm 1,718^{\mathrm{a}}$ \\
$\mathbf{J B}_{\mathbf{c}}$ & $6,6 \pm 0,071^{\mathrm{a}}$ & $93-98$ & $151,455 \pm 0,007^{\mathrm{a}}$ \\
$\mathbf{J C}_{\mathbf{n}}$ & $2,81 \pm 0,014^{\mathrm{b}}$ & $97-99$ & $58,74 \pm 1,117^{\mathrm{b}}$ \\
$\mathbf{J C}_{\mathbf{c}}$ & $2,05 \pm 0,071^{\mathrm{b}}$ & $98-99$ & $73,225 \pm 0,035^{\mathrm{b}}$
\end{tabular}

Dans une même colonne, les moyennes avec les mêmes lettres ne sont pas significativement différentes à 5\% 


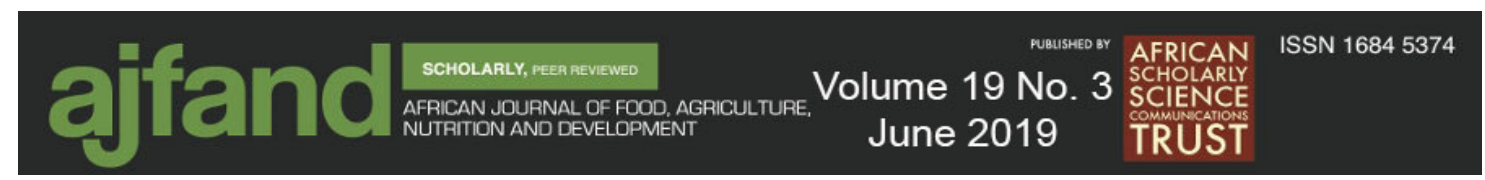

\section{RÉFÉRENCES BIBLIOGRAPHIQUES}

1. Talasila $\mathbf{U}$ and KB Shaik Quality, spoilage and preservation of cashew apple juice. $J$. Food Sci.Technol 2015; 52 (1): 54-62.

2. Honorato TL, Rabelo MC, Goncalves LRB, Pinto GAS and S Rodrigues Fermentation of cashew apple juice to produce high added value products. World $J$. Microbiol. Biotechnol 2007; 23:1409-1415.

3. Arora A and I Das Post-harvest processing technology for cashew apple- A review. Journal of Food Engineering 2016;194: 87-98.

4. Padonou SW, Houssou P, Karimou K, Todohoue MC, Olou D, Dossou J and GA Mensah Comparaison de quelques techniques d'extraction pour l'amélioration de la production et de la qualité du jus de pommes d'anacarde. Journal of Applied Biosciences 2015; 96: 9063 - 9071.

5. Dossou J, Boni $\mathbf{P}$ and $\mathbf{R}$ Cormier Clarification du jus de pomme de cajou. Quatre méthodes. Travaux de recherche appliquée. 2008. Anacardiuminfo.com consulté le 0903-16.

6. Dèdéhou SCAE, Dossou J, Ahohuendo B, Saidou A, Ahanchede A and MM Soumanou Optimisation de l'étape de la clarification du jus de pommes de cajou par l'amidon de riz et de manioc. J. Appl. Biosci. 2015; 95: 8989-9002.

7. AOAC. Official Methods of Analysis of the Association of Official Analytical Chemists International. 19 edition, Rockville, MD 20850, USA, 2012.

8. AFNOR T90 :105. Association Française de normalisation. Détermination de des matières en suspension. In : Recueil de normes françaises. Eaux : méthodes d'essais, 1979.

9. Dèdéhou ESCA, Dossou J and MM Soumanou Etude diagnostique des technologies de transformation de la pomme de cajou en jus au Bénin. International journal of Biological and Chemical Sciences 2015; 9(1): 371-387.

10. Akinwalé TO and O Aladesua Comparative study of physico-chemical properties and effect of different techniques on the quality of cashew juice from Brazilan and local varieties. Nig. J. Tree Crop Research 2001; 3 (1): 56-58.

11. Adou M, Tetchi FA, Gbane M, Kouassi NK and NG Amani Physicochemical characterization of cashew apple juice (Anacardiumoccidentale, L.) from Yamoussoukro (Côte d'Ivoire). Innovative Romanian Food Biotechnologie 2012; 11: $32-43$.

12. Brasil Leis Aprova os Regulamentos técnicos para fixação dos padrões de identidade e qualidade para polpas e sucos de frutas. Decretos, Instrução Normativa $n^{\circ} 1$ de 7 jan. 2000, di Ministério da Agricultura. Diário Oficial da União, Brasília, n. 6; 10 jan 2000. Seção I, 54-58. 


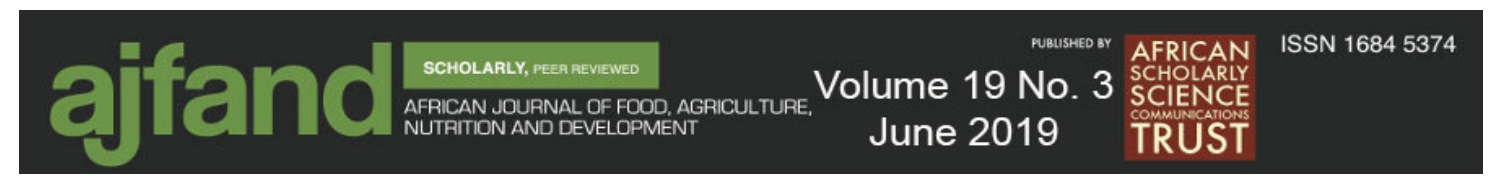

13. Egbekun MK and AO Otiri Changes in ascorbic acid contents in oranges and cashew apples with maturity. Ecology of Food and Nutrition 2010; 38: 275-284.

14. Drake SR and TA Eisele Influence of harvest date and controlled atmosphere storage delay on the color and quality of 'delicious' apples stored in a purge-type controlledatmosphere environment. Horticultural Technology 1994; 4: 260-263.

15. Oudot C La transformation des aliments. Castella-25, rue Mone-75005. 1999: 41-42.

16. Quoc AL, Fustier P, Couture R, Castaigne $\mathbf{F}$ and $\mathbf{J}$ Makhlouf Effect of various clarification agents on tannin removal, chemical composition and stability of cashew juice. Sciences des Aliments 1999; 18 (4):375-391.

17. Vivekanand V, Iyer $\mathbf{M}$ and $\mathbf{S}$ Ajlouni Clarification and Stability Enhancement of Pear Juice using Loose Nanofiltration. J Food Process Technol 2012; 3:162. doi:10.4172/2157-7110.1000162.

18. Damasceno LF, Fernandes FANM and ES Brito Evaluation and optimization of nonenzymatic browning of "cajuina" during thermal treatment. Brazilian Journal of Chemical Engineering. 2008; 25 (2): 313-320.

19. Food and Agriculture Organization/World Health Organization. Human vitamin and mineral requirements. Report of a Joint FAO/WHO Expert Consultation. FAO Food and Nutrition. Rome: WHO, 2001.

20. United States Department of Agriculture. Nutrition Database for Standard Reference. Washington, USDA, 2002.

21. Abreu F, Perez A M, Dornier M and M Reynes Potentialités de la microfiltration tangentielle sur membranes minérales pour la clarification du jus de pomme de cajou. EDP Science 2005; 60: 33-40.

22. Naka T, Kouakou MD, Soumaila D and KL Patrice Assessment of some bio-Chem. parameters of apple juices from two varieties as affected by three regions of Côte d'Ivoire. J. Adv. Agric. 2015; 5 (2): 6621 - 633.

23. Lowor ST and CK Agyente-Badu Mineral and proximate composition of cashew apple (Anarcadium occidentale L.): Juice from Northern Savannah, Forest and Coastal Savannah Regions in Ghana. Am. J. Food Technol 2009; 4: 154-161.

24. Agostini-Costa TS, Lima MV, Lima A, Aguiar MJ, de Lima JB and J Paiva Tanino em pedúnculos de caju: efeito de algumas variações genéticas e climáticas. B.Ceppa, Curitiba 2002; 20 (2): 267-278.

25. Jayalekshmy VG and PS John 'Sago' - a natural product for cashew apple juice clarification. Journal of Tropical Agriculture 2004; 42 (1-2): 67-68.

26. Talasila U, Vechalapu RR and KB Shaik Preservation and shelflife extension of cashew apple juice. Int. J. Food Safety 2011; 13: 275-280. 